\title{
PRACTICAL AND TACIT KNOWING AS A FOUNDATION OF INFORMATION SYSTEMS
}

\author{
John D. Haynes \\ Chair of Information Systems, Faculty of Business \\ UCOL, Palmerston North, New Zealand \\ j.haynes@ucol.ac.nz
}

\begin{abstract}
The importance of the human subject (and subjectivity) with its inherent capacity for intuition as a foundational concept for Information Systems is explored in this paper. The paper considers two philosophical contributions: (1) Michael Polanyi's distinction between Practical and Tacit Knowing, and (2) Rudolf Steiner's distinction between Intellectual Thinking and Intuitive Thinking. Intuition as it arises from subjectivity for an Information System is the key to determining the appropriate balance between practical and intuitive considerations necessary for the continued evolution of an Information System. Given this balance the theme of an Information System can emerge. Since an Information System is a social system bound to technology, it is the tacit capacities of an individual's subjectivity within an Information System that allow us to connect with the culture and the wider perspective of decisionmaking in an Information System.
\end{abstract}

\section{INTRODUCTION}

This paper is concerned with the relationship between human subjectivity and its capacity for intuition in relation to subjectivity and intuition within an Information System. It is contended that given that an Information System is a social system connected to technology, a fuller understanding of the Information System's social aspects, in particular the attributes of the humans that comprise the social system, will generate a better understanding of the Information System per se. In particular the work of two philosophers, Polanyi and Steiner, will be examined in the context of subjectivity and intuition. Early in the paper some aspects of Heidegger's work are cited in support of Polanyi's phenomenology and later in the paper brief aspects of the work of Habermas and Goethe are enlisted as support for Steiner's (and Polanyi's) perspective.

\section{PRACTICAL AND TACIT KNOWING}

It is helpful to begin an explication of Polanyi's distinction between Practical and Tacit knowing (also referred to as, respectively, Practical and Theoretical knowledge ${ }^{2}$ ) with a paradox that Polanyi $i^{3}$ himself discovered in Plato's work the Meno. Prosch (Prosch, 1986, p 96) mentions that:

To search for the solution to a problem, Plato told us there (the Meno), would seem to be absurd, since, if you know what you are looking for, then there is no problem. If you do not know what you are looking for, then you cannot expect to find anything. Polanyi maintained that this was a genuine paradox. Because, "to see a problem is to see something that is hidden. It is to have the intimation of the coherence of hitherto not comprehended particulars". Yet in spite of the apparent contradiction, involved in claiming to be able to see a problem, pointed out by Plato long ago, Polanyi noted that people have continued for two thousand years to see and solve many problems. What the Meno really shows, therefore, said Polanyi, is not that knowing is impossible (as indeed Plato also thought it did not show) but "that if all knowledge is explicit, i.e. capable of being clearly stated, then we cannot know a problem or look for a solution"4. Since we apparently do know good problems that can be solved, knowing a problem must be, he held, a kind of tacit knowledge, like the knowledge we have of a face or a class, a knowledge of which we cannot give a fully explicit account, but which nonetheless does exist.

Contained in the passage above is a key to unlocking Polanyi's distinction between Practical and Tacit knowing. In the above passage Polanyi is giving an example of Tacit knowing. For more abundant proof, Polanyi says precisely this in his Tacit Dimension (Polanyi, 1967, pp 22-23) "the kind of tacit knowledge that solves the paradox of the Meno consists in the intimation of something hidden, which we may yet discover". In my opinion, Polanyi clearly maintains a phenomenological stance for Tacit knowing, because for him, knowledge is implicit or hidden together with the recognition that it is the subject's intuition that at some level recognises the tacitness of the knowledge.

\footnotetext{
2 See (Polanyi 1967, pp 5,6).

3 I thank Frank Land for introducing me to the work of Michael Polanyi.

${ }^{4}$ Tacit Dimension, (Polanyi, 1967, p22)
} 
This recognition of the subject's intuition relates to both what the question touches upon and the knowledge contained in its subsequent answer. But it is the knowledge that is touched upon in the question of a problem that is hidden and therefore recognised as tacit, whereas the answer merely makes what is touched upon explicit. In other words, the subject recognises that knowledge or the path or the way to take to solve a problem is, in a very important sense, hidden already in the question that articulates the problem in the first place. For example, as is well known in Heidegger's work, Heidegger maintained that a question was far more important than its consequent answer. Heidegger considered this point from the perspective of phenomenology, that to ask a penetrating question is to already have come to an opinion on its answer. Often the very asking of a substantial question is like taking the lid off an almost pre-specified Pandora's box since a penetrating question also lays the paradigmatic groundwork, however complex, for its subsequent uncovering (or answering). But Polanyi saw the importance of a properly framed question for thinkers in general, or more precisely the values, assumptions and belief systems of individuals (in relation to their subjectivity) taken as human existence in general. We see this emphasis on individuals and their subjectivity again and again in Polanyi's work. And we see Polanyi's individual is - in a paradigm way - always grounded in an ethic of goodness or what benefits all individuals, as if the concern for all individuals allows each separate individual intuition to "flow" better. For example Webb notes (Webb, 1988, p 28) : " [in relation to] human existence for Michael Polanyi ... the individual is grounded in values and ethic, rather than in logic and reason". Why is it that ethics provides a different ground which is more fertile for intuition than to that of logic and reason for Polanyi? To uncover the hiddenness within this question we need to turn to Polanyi himself. In Personal Knowledge, (Polanyi, 1962, p 267) the answer, already implicit in the previous question, is revealed:

Our mind lives in action, and any attempt to specify its presuppositions produces a set of axioms which cannot tell us why we should accept them. ... Our basic beliefs are indubitable only in the sense that we believe them to be so. Otherwise they are not beliefs, but merely somebody's [some one else's] states of mind.

This then is our liberation from objectivism: to realise that we can voice our ultimate convictions only from within our convictions - from within the whole system of acceptances that are logically prior to any particular assertion of our own, prior to the holding of any particular piece of knowledge.

Ethics, we deduce from the above passages, that is, our own systems of accepted convictions, from within which we speak with conviction, or what I take to be an instance of a belief in our own beliefs, arise out of the our whole system of acceptances. Not from one piece of knowledge; not one reasoned bit; not one logical element or group of logical elements, but the whole system. So what Polanyi argues is the ground itself for the development of an individual's system of ethics comes from the whole system, not from one individual element. The power of an individual ethic arises because it is respectful of the whole system of acceptances. The emergence of an ethic for an individual - ethic being a belief in the good of things: action that both benefits the individual self and others in that one action - is not sidetracked by any individual desire. Nor is it sidetracked by any set of instances of selfgratification, but subsumes all of these desires and groups into the whole system of beliefs and takes its grounding from that synthesis. In this way an ethic develops independently of any logical or reasoned process. We do not condition our ethic by analysing it, rather our ethic arises out of the integration of all of our beliefs and desires. So our ethic is distilled and distinctly non-logical; non-reasoned and independent of "intellectual" processes. Our ethic retains its capacity for intuition because it is independent of reasoning and therefore emerges out of a deep sense of care. We are all born with this deep sense of care ${ }^{5}$. The quicker the emergence, I would suggest, the purer is the process of its being grounded and continuation for being grounded. One can imagine a paradigm case of an individual not being able to produce an ethic from which to base intuitions. Such a case would arise where certain obsessions and biases of self-gratification precluded a synthesis of the whole of the individual beliefs. A literal view of capitalism would be an example of such a bias.

Consider now why the question "why does ethics ground intuition and logic and reasoning does not" already contains the seeds of its own answer, or already provides a viewing of the hiddenness of the answer. Nor does it depend on the arrangement of words, I argue in Polanyian terms that a recognition of the hiddenness is still possible given further re-arrangements, such as:

Does intuition come from ethics or logic and reasoning?

I have indicated that for Polanyi ethics arises or emerges from a distillation of beliefs, and that such a distillation must have at some stage brought together both opposing and consistent beliefs. It is the critical nature of emergence that provides the clues to the recognition of hiddenness. Polanyi, as Webb notes (Webb, 1988, p 48) does "discuss a further major concept .. that of 'emergence'." But Webb further comments ( .. p 48) that Lonergan "makes better use of it than Polanyi succeeded in doing". In Philosophers of Consciousness, Webb (Webb, 1988, p 78) indicates

\footnotetext{
${ }^{5}$ I refer the reader to the work of Gary Zukav, in particular The Seat of the Soul (Rider Books, 1990) and the work of David Whyte in The Heart Aroused - Poetry and the Preservation of the Soul in Corporate America (Currency Doubleday, New York, 1994) for an explication of a human's deep sense of care in the context of organisations - which is outside the scope of this paper.
} 
Lonergan's extension to Polanyi's concept of emergence was the recognition that things that are viewed as recognised as having hidden qualities have "proportionate beings ... ( and have) .. 'parallel structures' to our knowing". Webb further comments of Lonergan's contribution (..p 79) that by "proportionate being, he says, is intrinsically intelligible because it is precisely that which we are able to inquire into". In other words there is something in the question itself that triggers a response in the system of beliefs. What does this mean? It means that in asking a question for which we can recognise a hidden answer, we are putting forward a question that has already been asked ${ }^{6}$ at an unconscious level. At this unconscious level the asking has been "sorted out"? and we, as an individual, are now putting that question forward for the conscious level of our being to apprehend. This is the way Tacit knowing is both recognised as hidden and how it makes itself manifest as a process of uncovering what is hidden.

A practical way of seeing the point that $I$ am making is in relation to the example that $I$ have explicated as a resolution to Polanyi's paradox of the Meno, which is both an example of Tacit knowing and - by the resolution of the paradox - a recognition ${ }^{8}$ of it in action. Perhaps another way of seeing the concept of emergence is to consider a situation where rational thinking is argued not to apply. Flemons in Completing Distinctions notes a situation based on Taoist principles where rational knowledge is argued to be of no use. Consider the following passage (Flemons, 1991, p 70):

If we are up against a mystery, then we dare act only on the most modest assumptions. The modern scientific program has held that we must act on the basis of knowledge .. but if we are up against a mystery, then knowledge is relatively small, and the ancient program is the right one: act on the basis of ignorance.

In other words, from the above passage the meaning is that in acting from ignorance we are not contaminating our intuition $^{10}$ with our rational knowledge ${ }^{11}$. And in this strict sense ignorance can ultimately know more than knowledge.

On the other hand, practical knowledge for Polanyi does not have any hidden aspects to it. In the Tacit Dimension (Polanyi, 1967, p 32, 33), Polanyi states that things for which practical knowledge is appropriate are "less real" than things for which Tacit knowing applies. He gives the example of cobblestones as being more real (than tacitly known things) in the sense of being more tangible, but less real in the sense that they have no hiddenness to them. Polanyi's view of the objects of practical knowledge align fairly closely with Heidegger's concept of a thing. Joseph Kocklemans in his book On the Truth of Being - Reflections on Heidegger's Later Philosophy, had this to say about a third conception of a Heideggerian thing that I conclude is close to Polanyi's object of practical knowledge (Kockelmans, 1984, pp 175,176):

The thing is nothing but formed matter; and this conception also holds good for both natural and man made things. This conception accounts for the thingly element we find in every work of art.

If an object then is "thingly" it exhibits an absence of hiddenness if and only if it is the pure "thingliness" of the object that we are considering. Under such circumstances it is a candidate for being an object of practical knowledge. Examples of such practical knowledge would be diagrams entailing information as distinct from the mathematics behind that information (the mathematical process of producing that information).

\section{PRACTICAL AND TACIT INFORMATION SYSTEMS}

An Information System is a social system bound to technology. The practical knowledge of an Information System includes its technological elements and all other considerations that are conducive to logical analysis. But insofar as it is a social system it carries the power inherent in the humanity that comprises that social system. The upshot of Polanyi's position in relation to human subjectivity is that its capacity for intuition based on Tacit knowing allows it to recognise the difference between practical and tacit considerations. Without the tacit capacities of subjectivity we would, as a human component of an Information System, be nothing but practical (or machine-like) in our decisionmaking. By providing a thorough-going conception of the features and power of Tacit knowing Polanyi allows an Information System to see itself in terms of its own evolvement. The Polanyian concepts of hiddenness, emergence and "tacitness grounded in ethics rather than logic" allows us to see a wider perspective for an Information System, that like a human being an Information System is founded on more than the components that make it up.

\footnotetext{
${ }^{6}$ To ask a question at the unconscious level is to virtually answer it, hence my expansion in the next sentence of the need for the asking to be "sorted out".

${ }^{7}$ Or more precisely placed within the context of the synthesis of the whole system of beliefs.

${ }^{8}$ That is, the "tacit knowing" of the reader.

${ }^{9}$ Quoted from Wendel Berry, in Home Economics, North Point Press, San Francisco, 1987, pp 4-5.

${ }^{10}$ Which, incidentally, literally means inner-teaching.

"Hence, from footnote 9, we may construe this as outer-teaching.
} 


\section{INTELLECTUAL THINKING AND INTUITIVE THINKING}

It seems apt to cross from Polanyi to Steiner with a quotation from Polanyi on Goethe, if only because Steiner was greatly preoccupied with the work of Goethe and Polanyi was certainly sympathetic with Goethe's general views. Polanyi made the point that (Polanyi, 1967, pp 79-80):

The degree of originality any particular scientist trusts himself to possess should thus determine the range which he will venture to tackle and hence also the range of information which he will unquestioningly accept. Goethe wrote that the master proves himself by his restraint.

For Polanyi, originality then, inter alia, comes from an ability to be able to discriminate "within" information. I suggest that such discrimination is evidence of selecting only that which is appropriate to the theme of the inquiry. I suggest that if a theme can be recognised then meaning can be perceived. The theme of the inquiry is a key to Goethe's (by Bortoft ${ }^{12}$ ) and Steiner's distinction between Intellectual and Intuitive thinking. Such a key can be clearly discerned as be-fitting of Bortoft's Goethe in the following passage (Bortoft, 1996, p 57):

Discovery in Science is always a perception of meaning, and it could not be otherwise. The essence of a discovery is therefore in the nonempirical factor in cognition. The recognition that meaning is a primary datum of cognitive experience brings a considerable simplification to the philosophy of science. Of course, the meaning in question may be several stages removed from the meaning in everyday cognition, and at a much more comprehensive level.

And in Steiner too, we see the following passage echoes the nature of meaning and theme in relation to cognitive experiences of the "higher states"13 and the "everyday states"14 (Steiner, 1995, pp 182-183):

Moral efficacy depends on knowledge of the phenomenal world with which one is dealing. This knowledge must therefore be sought in a branch of general scientific knowledge. Hence, along with the faculty for moral ideas and imagination, moral action presupposes the capacity to transform the world of percepts without interrupting its coherence in natural law.

By "moral efficacy" Steiner means a realisation (making real; bringing into being) of meaning, or as he says himself (.. p 182):

Free spirits need moral imagination to realise their ideas and make them effective ... To turn a mental picture into a reality, moral imagination must set to work in a specific field of percepts. Human action does not create percepts, it recasts already existing percepts and gives them a new form. To be able to transform a specific perceptual object or group of objects in accordance with a moral mental picture, one must have understood the laws of the perceptual picture to which one wants to give new form or new direction.

A free spirit to Steiner is one who is not limited by what others may think nor by what his or her ego dictates. In this way the free spirit is unencumbered, and, given the opportunity to develop an imagination (as the consequence of a special form of action), can, theoretically, begin to draw any contradictory idea or underpinning together to consider. The results of such considerations are then filtered through the "moral" imagination, which for Steiner, is always mindful of the good of the individual and the good of all individuals simultaneously. The moral imaginer is therefore able to make real (real-ise) his or her ideas more effectively because they have been given flight by the Steinerian "moral" dimension. The upshot for the Steinerian system is that a person living according to the practices of a moral imagination, draws to him/herself a community of imagination (although he would not use these terms). It is the very comfort and support of this community that provides the ground for making the fruits of moral imagination more effective. The notion of "percepts" (in the next section) sheds more light on the inner process of the thinking of a moral imaginer and indeed Tacit knowing itself.

\section{The Notion of Percepts}

The Bortoftian comment that "the essence of discovery is in the nonempirical factor of cognition" and the Steinerian remark that "human action does not create percepts, it recasts already existing percepts and gives them a new form" are a conceptual collage of each other. Both use the same concepts but they are juggled in different ways and the effect is that each conception explains the other. When we consider that a percept is the mental result or product of the act of perception, I argue that we are very much reminded of the unconscious side of Tacit knowing (as mentioned above) and how it makes itself concretely manifest and thereby uncovers what is hidden. So, in this sense, human action does not create the unconscious side of Tacit knowing - that is already given - "it recasts" what is hidden as existing percepts and gives those percepts a new form. The hidden percept is what Bortoft means by the nonempirical factor of cognition. But it is precisely the essence of discovery that resides in the hidden side of

\footnotetext{
${ }^{12}$ Who, himself, was a Steinerian.

13 "Higher states" refer to holistic modes of thinking, for example, composing something original.

14 "Everyday" states refer to practical considerations, such as survival needs.
} 
cognition and the concretisation of that particular discovery (or any particular discovery hidden in cognition) is the new form that becomes the empirical result of cognition. Furthermore, it is the empirical result of cognition that is then perceived as having meaning, because what perceives it is the nonempirical factor in the observer's cognition and it is this realisation that, for example, "brings a considerable simplification to the philosophy of science".

To conceptually reconfigure Bortoft's and Steiner's remarks we have:

* Human action does not create the essence of discovery.

* Why? Because the essence of discovery already lies in the creation of percepts.

* The creation of percepts is a constantly occurring nonempirical factor of cognition.

* Human action recasts the essence of discovery and gives it a new visible form.

The result appears to be innocent enough: in order to realise a certain discovery that one has made, one needs to perform a certain action that makes what is implicit as a percept explicit as a new concrete appearance of that form. But Bortoft and Steiner have a more powerful and extensive vista in mind. This seems to be that a special form of action is more powerful than thought itself. The implication is that thought is somehow always there; always in the process of coming to percepts; always coming to the essences of various discoveries. Whereas the differences in mankind are revealed in the kinds of actions that they perform that consequently enable them to uncover those percepts; that express an ability to give them a "new form" as a real appearance. So, differences in the abilities of (at least normal) humans are, therefore, not intellectual per se, but are a direct consequence of the active physical process by which humans access the percepts of their cognition; access the mental result or product of their intuitions.

For Steiner and Bortoft, I submit, thought is subservient to a special form of action. In suggesting a distinction between intellectual thinking and intuitive thinking, it is all forms of intellectual thinking that fall short of ${ }^{15}$ the pervasive creative power of intuitive thinking.

\section{Eastern Thinking}

This is also born out by a long tradition in Eastern cultures. As Joseph Bogen notes (Bogen, 1973, p 124), Hindu and Buddhist teaching distinguishes intellect (buddhi) from mind (manas) as "rational thought" (intellectual) and "integral thought" (intuitive) respectively. "Rational thought" is considered to be far inferior to "integral thought".

\section{Habermas}

It seems Habermas, for example, would support Intuitive thinking over Rational thinking, even though Habermas is taken to be one of the fathers of rational discourse in the more recent Information Systems literature (see (Hirschheim \& Klein, 1996)). We see Habermas extending rationality to a concept (i.e. beyond rationality) and transcending it even to consciousness, in the following points made by Thomas McCarthy (McCarthy, 1978, pp 272, 273):

[Habermas's] theory of communicative competence is a new approach to a familiar task: to articulate and expound an expanded conception of rationality ... the transcendental critiques of language transcends that of consciousness.

From McCarthy we can see that Habermas's project is distinctly not a philosophy of science approach. It is clear that Habermas's interests are far more towards phenomenology and hermeneutics because Habermas, like Polanyi and Steiner (and Goethe), is interested in an "expanded conception of rationality". It is often the case that phenomenology is not associated with Habermas. However, as further evidence, I think the following passage from Martin Jay referring first to Benjamin and then to a quotation from Habermas, places Habermas at the very least in the phenomenological tradition (Jay, 1985, p 129):

What makes this type of expression and mimesis so attractive for Benjamin is its priority to the break between subject and object; in other words, that Schillerian motif of reconciliation ... "As odd as this mimetic [Benjamin's] theory of language sounds", he [Habermas] contended, Benjamin is right in assuming that the oldest semantic stratum is that of expression ... with the meanings that comprise this potential, human beings interpret the world in terms of their own needs and create thereby a net of correspondences." $" 16$

One of the interesting consequences of Habermas wanting to extend the concept of Western rationality, is the implication that existing Western rationality cannot maintain itself as it is. Or further, that it needs to be replaced altogether. Personally I do not think that Western rationality is finished. I agree with Yount (following Marsh) that it can be redeemed dialectically. "Is the tradition of modern Western rationality simply to be overcome or is it to be redeemed dialectically"; "Could it be, Pythagoreans, that this irrationality belongs to the hypotenuse, and not the

\footnotetext{
${ }^{15}$ But are nevertheless critical to the functioning of human beings.

${ }^{16}$ Habermas, "Consciousness-Raising or Redemptive Criticism", pp 48-49 (no reference provided by Jay).
} 
geometer?" (Yount, 1992, p 29). In other words, Western rationality, which entails the inclusion of Western conceptions of Information Systems, needs to be redeemed dialectically.

Goethe

Now consider the implications of the role of intellectual thinking in the following quotation from Bortoft (Bortoft, 1996, p 58):

The recognition of the active role of the intellectual mind gave rise to a philosophy of science which maintained the view that a scientific theory is only a framework which we construct for holding the facts together for our own convenience - where in this case it is believed that the facts themselves are perceived entirely by the senses, independently of the theory.

The above quote shows why Bortoft sees obvious deficiencies in elevating intellectual thinking over intuitive thinking. The quotation also illustrates how an intellectual fact-based approach could not even conceive of the possibility of another form of thinking, such as the intuitive, because there is no ground from which such a possibility could spring. In the following passage Bortoft considers the implications of the intuitive approach as a practical alternative, while also establishing some of the implications of the deficiencies in an intellectual approach (Bortoft, 1996, pp 66-67):

[The] psychological interpretation of Goethe's way of access to nature in terms of deautomatization is reinforced by considering the subjective experience of the procedure. Through trying to direct attention to the act of looking, we can experience for ourselves the gap which there is between our habitual awareness and the direct experience of what is there in front of us. It is only when the hiatus in experience is overcome that we realise how little we usually perceive directly of the concrete detail of the particular. We usually classify verbally and experience just a vague generality. A striking feature of this attempt to give attention to active looking and exact sensorial imagination is how much subjective resistance it can set up in a person. This in itself is an indication that the orientation which it calls for is towards deautomatization or dishabituation. This subjective effect is an instance of the psychological inertia which has to be matched by a person's own activity if the state of his or her awareness is to change, just as the inertia of a material body has to be matched by a force if its state of motion is to be changed.

When consciousness is thus restructured into an organ of holistic perception, the mind functions intuitively instead of intellectually. There is a lot of confusion and misunderstanding about intuition, as if it were something intangible and mysterious. But in fact it is a very clear and precise notion. Omstein defines intuition as "knowledge without recourse to interference"17. He links it with a simultaneous perception of the whole, whereas the logical or rational mode of knowledge "involves an analysis into discrete elements sequentially (inferentially) linked"18. He connects the intuitive mind with the holistic mode of consciousness - as the intellectual mind is linked with the analytical mode. Thus intuition is connected with a change of consciousness, and moreover in a way which can be made quite precise and not just left vague. It now follows that Goethe's procedures are practical exercises for educating the mind to function intuitively instead of intellectually, leading to a science which is intuitive instead of organised intellectually.

What are the practical exercises that Goethe speaks of above? Bortoft tells us (Bortoft, 1996, p 75) that Goethe:

Learned to read [a] plant in terms of itself, so that the plant becomes its own language, and secondly how it is possible to read the language of animal form so that the animal becomes its own explanation. In view of this, Goethe's intuitive way of science can be recognised as a concrete illustration of Gadamer's principle of universal hermeneutics that "being that can be understood is language"19. The philosophy of Goethe's science can therefore be identified more precisely as the hermeneutic phenomenology of nature.

The difficulty between Goethe's phenomenological way and the mainstream of mathematical physics from Newton onwards was summarized memorably and concisely by Cassirer: "The mathematical formula strives to make phenomena calculable, that of Goethe to make them visible"20.

It is now possible to discuss the special form of action that Bortoft and Steiner indicate is essential for engaging intuitive thinking. In phenomenology we move with the concepts that are pointed to in a written piece of phenomenological literature. The same sense of movement applies as a project for Bortoft and Steiner. Except for Bortoft and Steiner it is a theme that is being actively looked for, without any interference of thinking about what

\footnotetext{
${ }^{17}$ Robert E. Ornstein, The Mind Field, Octagon Press, London, 1983, p 24.

${ }^{18}$ Ibid, p 26.

${ }^{19}$ (Gadamer, 1975, p 474)

${ }^{20}$ A.G.F. Gode von Aesch, Natural Science in German Romanticism, Columbia University German Studies, $1941, \mathrm{p} 74$.
} 
we are looking for, we simply let the theme, as it were, speak to us and we engage with that "speaking". In the speaking we hear the "language" that Gadamer speaks of as the "being" (Gadamer, 1975, p 474).

For Bortoft and Steiner it is the theme of a phenomenon that speaks to us in the language of thought; a language of concepts. The special action is subjective but in order to remain special it is not interfered with by any kind of analysis; by any kind of intellectual thinking about what is being performed. We simply move with whatever is presented to us. Goethe gives examples of plants and animals forms becoming their own speaking or language, but any living matter or manner of a work by man or woman potentially holds conceptual themes that can be comprehended in a like manner. Perhaps an example from architecture will help. A doorway for any type of building can be appreciated for itself as announcing a forthcoming interior. That announcement is implicit until someone moves through the doorway. The more important the building, the more effective should be the announcement (note the case of certain religious buildings where one is forced - by the restriction in the height of the doorway - to stoop or bow one's head to enter). But if a person was preoccupied with some matter (other than being with the experience of the doorway) while walking through the doorway, the effect of the announcement of the forthcoming interior would be lost - or at the very least reduced. If the person was engaging in moving through the doorway; experiencing it as a shift in space and experiencing the design of the architecture, then the implicit announcement "hidden" in that design would announce itself in an explicit conceptual way - as an experience - to that person.

Consider another example from the area of interior design. As a person gets older often the memories of when a familiar article of clothing was last worn begins to fade or even the memory becomes unimportant. Imagine then a walk-in wardrobe that contained pictures of that person in happy times wearing articles of clothing that were still available for wear in that wardrobe. The possibility that I am suggesting is that with prompts or cues even dressing in familiar clothes can be an experience of uninterrupted subjectivity: the clothing can potentially "speak" to the wearer of past experiences that the wearer can experience via those clothes. Perhaps the photos can be placed outside the walk-in wardrobe. In this case the person in question might go into the wardrobe mindful of some memory and it could be the memory that speaks to the person of the choice of clothing to wear.

\section{IMPLICATIONS FOR INFORMATION SYSTEMS}

In all three cases above, I try to convey (1) a sense of being at ease with what is being done or experienced, and (2) exploring a set of circumstances which allow for a heightening of awareness of what is being experienced as it is being experienced (and thereby staying in that experience). It is circumstances like these that Bortoft and Steiner argue - although they do not use these examples - set in train intuitive thinking. The more often we stay in an experience of an outside phenomenon and let that experience "speak" to us, the better we are able to access our primary internal phenomenon, namely our percepts, thereby letting the fruits of our intuitive abilities, also "speak to us". The same phenomenon occurs in an Information System, if we allow logical analyses of an Information System to dominate our thinking then we are not free to explore other avenues of expanding the Information System. One way of appreciating a sense of expanding our consciousness in relation to an Information System is to consider the concept of the culture of an Information System. One clear way in which employees fail to contribute their full potential as a cohesive social unit to the Information System is where individuals lack a sense of belonging to the Information System. This feeling of an absence of belonging leads to a fragmentation of the social unit and consequently diminishes the potential contribution each employee can make. It needs no reminding that corporations lose large portions of their potential net profits each year as a result of employees who do not understand how they "fit" into the "structure" of a corporation. This alienation is passed on to customers of the corporation through the inept actions of the alienated employee. By understanding intuitive thinking a culture can be established and allowed to emerge in an Information System. The culture of an Information System is the way in which the theme of an Information System (the particular products which are developed in relation to its inherent social system) makes itself manifest.

\section{CONCLUSION}

For both Polanyi and Steiner the implications of the above distinctions for an Information System point to the view that an Information System is potentially thematic. That is, each Information System, if it is allowed to develop as a culture, has its own theme. For Polanyi Practical Knowing can never be any more than collections of facts, whereas Tacit Knowing is able to comprehend both itself and Practical Knowing, which means that Tacit Knowing comprehends the essence or themes of things. For Steiner Intuitive Thinking is the language of thought or concepts and as such is distinctly thematically based. The decision-makers of an Information System will not discover the theme of an Information System in a logical way; no amount of reporting on profits and losses or efficiencies and failures will discover that theme. The theme is only apprehended by intuition. The absence of the discovery of the theme of a particular Information System also entails the absence of an effective capacity to determine how that 
Information System will react to powerful changes. In short, without a comprehension of its own theme an Information System cannot effectively evolve.

\section{REFERENCES}

Bogen, Joseph E. (1973) The Other Side of the Brain: An Appositional Mind, in The Nature of Human Consciousness - a Book of Readings Edited by Ornstein, Robert E , W.H. Freeman and Co. San Francisco, pp 101-125.

Bortoft, Henri (1996) The Wholeness of Nature - Goethe's Way Toward a Science of Conscious Participation in Nature, Lindisfarne Press, Hudson NY, USA.

Flemons, D.G. (1991) Completing Distinctions, Shambhala Books, Boston, USA.

Gadamer, H-G. (1975) Truth and Method, translated and edited by Barden, G and Cumming, J, Seabury Press, New York.

Hirschheim, Rudy and The Rationality of Value Choices in Information Systems Klein, Heinz K. (1996)

Development, Foundations of Information Systems, Electronic Journal, http://www.cba.uh.edu/ parks/fis/fis.htm

Jay, Martin (1985) Habermas and Modemism, in Habermas and Modernity. Bernstein, R.J. (Ed), The MIT Press, Cambridge, Mass, USA, pp 126- 139.

Kockelmans, Joseph, J(1984) On the Truth of Being - Reflections on Heidegger's Later Philosophy. Indiana University Press Bloomington, USA.

McCarthy, Thomas (1978) The Critical Theory of Gurgen Habermas. MIT Press Cambridge, Mass, USA.

Polanyi, Michael (1962) Personal Knowledge - Towards a Post Critical Philosophy. The University of Chicago Press, USA.

Polanyi, Michael (1967) The Tacit Dimension. Anchor Books, Double Day and Company, Garden City, New York.

Prosch, Harry (1986) Michael Polanyi - A Critical Exposition. State University of New York Press, USA.

Steiner, Rudolf (1995) Intuitive Thinking as a Spiritual Path: A Philosophy of Freedom, Anthroposophic Press, Hudson, NY, USA.

Webb, Eugene (1988) Philosophers of Consciousness - Polanyi, et al. University of Washington Press. USA.

Yount, Mark (1992)Uncapitalising on Radical Hermeneutics, Modernity and its Discontents, Fordham University Press, USA, pp 23-44. 